PROCEEDINGS OF THE

AMERICAN MATHEMATICAL SOCIETY

Volume 132, Number 6, Pages 1605-1612

S 0002-9939(03)07242-3

Article electronically published on October 21, 2003

\title{
MINIMAL POLYNOMIALS OF ELEMENTS OF ORDER $p$ IN $p$-MODULAR PROJECTIVE REPRESENTATIONS OF ALTERNATING GROUPS
}

\author{
A. S. KLESHCHEV AND A. E. ZALESSKI
}

(Communicated by Stephen D. Smith)

\begin{abstract}
Let $F$ be an algebraically closed field of characteristic $p>0$ and let $G$ be a quasi-simple group with $G / Z(G) \cong A_{n}$. We describe the minimal polynomials of elements of order $p$ in irreducible representations of $G$ over $F$. If $p=2$, we determine the minimal polynomials of elements of order 4 in 2-modular irreducible representations of $A_{n}, S_{n}, 3 \cdot A_{6}, 3 \cdot S_{6}, 3 \cdot A_{7}$, and $3 \cdot S_{7}$.
\end{abstract}

\section{INTRODUCTION}

Throughout the paper, $F$ is an algebraically closed field of characteristic $p>0$ and all representations are $F$-representations unless otherwise stated. Let $A_{n}$ and $S_{n}$ denote the alternating and symmetric groups on $n$ letters. We always assume that $n \geq 5$. Let $G$ be a quasi-simple group with $G / Z(G) \cong A_{n}$, and let

$$
\pi: G \rightarrow A_{n}
$$

be the natural projection. Thus $G$ is one of the following groups: $A_{n}, \tilde{A}_{n}:=2 \cdot A_{n}$, $k \cdot A_{6}$, or $k \cdot A_{7}$ for $k=3,6$.

Our goal is to determine the minimal polynomials of the elements $g \in G$ of order $p$ in the irreducible representations of $G$. Minimal polynomials of such elements are always of the form $(x-1)^{d}$ for some $d \leq p$, and we determine all configurations where $d<p$.

Theorem 1.1. Let $G$ be a quasi-simple group with $G / Z(G) \cong A_{n}$, let $g \in G \backslash Z(G)$ be an element of order $p$, and let $\phi$ be a faithful irreducible representation of $G$ over $F$. Then the degree $d$ of the minimal polynomial of $\phi(g)$ is less than $p$ if and only if one of the following happens:

(i) $\pi(g)$ is a product of two 3 -cycles, $G=\tilde{A}_{6}, p=3$, and $\phi$ is a basic spin representation of dimension 2 .

(ii) $\pi(g)$ is a p-cycle and one of the following holds:

(a) $G=A_{p}$, and $\phi$ is the "natural" representation of dimension $p-2$;

(b) $G=\tilde{A}_{n}, p=3$ or 5 , and $\phi$ is a basic spin representation;

(c) $G=3 \cdot A_{7}$ or $6 \cdot A_{7}, p=7$, and $\operatorname{dim} \phi=6$;

(d) $G=\tilde{A}_{7}, p=7$, and $\operatorname{dim} \phi=4$;

(e) $G=\tilde{A}_{5}, p=5$, and $\operatorname{dim} \phi=4$;

Received by the editors November 18, 2002 and, in revised form, February 19, 2003.

2000 Mathematics Subject Classification. Primary 20C30; Secondary 20C20, 20 D06.

(C)2003 American Mathematical Society 
(f) $G=3 \cdot A_{6}$ or $3 \cdot A_{7}, p=5$, and $\operatorname{dim} \phi=3$.

Moreover, $d=p-1$ in the case (ii)(b) above, and $d=\operatorname{dim} \phi$ in the remaining exceptional cases.

In particular, we see that there are two "reasons" for the minimal polynomial of an element of order $p$ to have degree less than $p$ in an irreducible representation of $G$. One is trivial - the dimension of our representation might be less than $p$. The other is less obvious $-p=3$ or 5 and the representation is a basic spin representation (these representations are known to be a source of many counterexamples and are pretty well-understood). We note that the degrees of the basic representations of $\tilde{A}_{n}$ in prime characteristic may differ from those in zero characteristic; see Lemma 2.6 below.

In the proofs we only have to deal with the case $p>3$, since the case $p=3$ of Theorem 1.1 has recently been settled by Chermak 3].

Obviously, the case $p=2$ is trivial for elements of order 2. However, a version of the question for $g \in G$ of order 4 is of essential interest. Of course, when $p=2$ we do not need to deal with two-fold coverings. However, the case $G=S_{n}$ does not automatically reduce to $A_{n}$ since $g$ may not belong to $A_{n}$. So we consider $S_{n}$ as well.

Theorem 1.2. Let $p=2, n \geq 5, G \in\left\{A_{n}, S_{n}, 3 \cdot A_{6}, 3 \cdot S_{6}, 3 \cdot A_{7}, 3 \cdot S_{7}\right\}, g \in G$ be an element of order 4 , and let $\phi$ be a faithful irreducible representation of $G$ over $F$. Then the degree $d$ of the minimal polynomial of $\phi(g)$ is less than 4 if and only if $d=3$ and one of the following happens:

(a) $g$ is of cycle type $(4,2)$, and either $G \cong 3 \cdot A_{6}, \operatorname{dim} \phi=3$ or $G \cong 3 \cdot S_{6}$, $\operatorname{dim} \phi=6$;

(b) $G \cong A_{8} \cong S L(4,2), g$ is of cycle type $(4,4)$, and $\phi$ is either the natural representation of $S L(4,2)$, or its dual, or its exterior square;

(c) $G \cong S_{8}, g$ is of cycle type $(4,4)$, and $\operatorname{dim} \phi=8$ or 6 .

\section{Preliminaries}

If $M$ is a matrix, we denote by $\operatorname{deg} M$ the degree of the minimal polynomial of $M$ and by Jord $M$ the Jordan normal form of $M$ (defined up to the ordering of Jordan blocks). The Jordan block of size $k$ with eigenvalue 1 is denoted by $J_{k}$. The symbol $\operatorname{diag}\left(a_{1}, \ldots, a_{k}\right)$ denotes the block-diagonal matrix with square matrices $a_{1}, \ldots, a_{k}$ along the diagonal.

If $G$ is any group, we denote by $1_{G}$ the trivial $F G$-module (or the corresponding representation). If $M$ is an $F G$-module (resp. $\phi: G \rightarrow G L(M)$ is a representation of $G$ ), and $H<G$ is a subgroup, then $M \mid H$ (resp. $\phi \mid H$ ) stands for the restriction of $M$ (resp. $\phi)$ to $H$.

We record the following obvious fact.

Lemma 2.1. Let $G$ be a finite group and $g \in G$. If $\rho$ and $\phi$ are representations of $G$ such that $\rho$ is a subfactor of $\phi$, then $\operatorname{deg} \rho(g) \leq \operatorname{deg} \phi(g)$.

Let $m<n$. Throughout the paper we will often consider $S_{m}$ as a subgroup of $S_{n}, \tilde{A}_{m}$ as a subgroup of $\tilde{A}_{n}$, etc. Unless otherwise stated, the embeddings are assumed to be natural, i.e., the subgroup acts on the first $m$ letters.

Now, let $G=A_{n}$ or $S_{n}$. We will refer to the nontrivial composition factor of the natural $n$-dimensional permutation $F G$-module as the natural irreducible module 
and denote it by $E_{n}$. Denote by $\varepsilon_{n}$ the corresponding representation. We have $\operatorname{dim} \varepsilon_{n}=n-2$ if $p \mid n$ and $\operatorname{dim} \varepsilon_{n}=n-1$ otherwise.

Lemma 2.2. Let $G=S_{n}$ or $A_{n}$ and let $g \in G$ be an element of order $p$. Then the degree $d$ of the minimal polynomial of $\varepsilon_{n}(g)$ is $p$, unless $n=p$, in which case $d=p-2$.

Proof. An easy explicit calculation (see, e.g., [8, Lemmas 2.1 and 2.2]).

Let $1_{S_{n}}$ be the sign module over $F S_{n}$, and set $E_{n}^{-}=E_{n} \otimes 1_{S_{n}}^{-}$. Define

$$
\mathcal{E}_{n}:=\left\{1_{S_{n}}, 1_{S_{n}}, E_{n}, E_{n}^{-}\right\} .
$$

If $\lambda$ is a $p$-regular partition, $D^{\lambda}$ denotes the irreducible $F S_{n}$-module corresponding to $\lambda$; see [6]. The following is a useful inductive characterization of the $F S_{n}$-modules from $\mathcal{E}_{n}$.

Proposition 2.3. Let $n \geq 6$ and let $D$ be an irreducible $F S_{n}$-module. Suppose that all composition factors of the restriction $D \mid S_{n-1}$ belong to $\mathcal{E}_{n-1}$. Then $D \in$ $\mathcal{E}_{n}$, unless $n=6, p=3$ and $D \in\left\{D^{(4,2)}, D^{\left(2^{2}, 1^{2}\right)}\right\}$, or $n=6, p=5$ and $D \in$ $\left\{D^{\left(4,1^{2}\right)}, D^{\left(3,1^{3}\right)}\right\}$.

Proof. By tensoring with $1_{S_{n}}^{-}$if necessary, we may assume that $1_{S_{n-1}}$ or $E_{n-1}$ occurs in the socle of $D \mid S_{n-1}$. Then it follows from [7, Theorem 0.5] that either $D \in \mathcal{E}_{n}$ or $D \in\left\{D^{(n-2,2)}, D^{\left(n-2,1^{2}\right)}\right\}$. However, by [7, Theorem $0.4($ ii $\left.)\right], D^{(n-2,2)} \mid S_{n-1}$ contains $D^{(n-3,2)}$ as a composition factor, and $D^{(n-3,2)} \notin \mathcal{E}_{n-1}$ unless $n=6$ and $p=3$. Similarly, $D^{\left(n-2,1^{2}\right)} \mid S_{n-1}$ contains $D^{\left(n-3,1^{2}\right)}$ as a composition factor and $D^{\left(n-3,1^{2}\right)} \notin \mathcal{E}_{n-1}$ unless $n=6$ and $p=5$.

Corollary 2.4. Let $n \geq 7$, and let $V$ be an irreducible $F A_{n}$-module such that all composition factors of the restriction $V \mid A_{n-1}$ belong to $\left\{1_{A_{n-1}}, E_{n-1}\right\}$. Then $V \in\left\{1_{A_{n}}, E_{n}\right\}$.

Proof. Follows from Clifford theory and Proposition 2.3.

We need the following result of Benson in characteristic 2:

Lemma 2.5 ([1]). Let $\lambda=\left(\lambda_{1}, \lambda_{2}, \ldots\right)$ be a 2-regular partition. Then $D^{\lambda} \mid A_{n}$ splits as a direct sum of two non-equivalent irreducible $F A_{n}$-modules if and only if for all $j$ with $\lambda_{2 j-1}>0$ we have $\lambda_{2 j-1}-\lambda_{2 j}=1$ or 2 , and $\lambda_{2 j-1}+\lambda_{2 j} \equiv 2(\bmod 4)$. Otherwise, $D^{\lambda} \mid A_{n}$ is irreducible.

Let $\tilde{S}_{n}$ denote a (nontrivial) two-fold central cover of $S_{n}$. Of course, $\tilde{A}_{n}$ is a subgroup in $\tilde{S}_{n}$ of index 2 . The group $\tilde{S}_{n}$ has (one or two) remarkable complex representations called basic (spin) representations. These can be characterized as its faithful complex representations of minimal degree and can be constructed using Clifford algebras. A basic spin representation can also be defined as an irreducible representation of $\tilde{S}_{n}$ whose character is labelled by the partition $(n)$ in Schur's parametrization of irreducible characters. The degree of a basic representation of $\tilde{S}_{n}$ is $2^{(n-1) / 2}$ if $n$ is odd, and $2^{(n-2) / 2}$ if $n$ is even. On restriction to $\tilde{A}_{n}$, basic representations remain irreducible if $n$ is even and split as a direct sum of two non-equivalent irreducibles if $n$ is odd. In both cases the corresponding complex representations of $\tilde{A}_{n}$ are also called basic. 
Finally, for both $\tilde{S}_{n}$ and $\tilde{A}_{n}$, every irreducible constituent of Brauer reduction of a basic representation modulo $p$ is called a (modular) basic representation. Dimensions of modular basic representations of $\tilde{S}_{n}$ have been determined by Wales [11. For $p>2$, these are the same as for complex representations, unless $p$ divides $n$, in which case they are twice as small. Moreover, in [11 Table III], Wales provides complete information concerning tensoring basic modular representations with sign, from which the dimensions of basic modular representations of $\tilde{A}_{n}$ also follow, at least for $p>2$. If $p=2$, one can use Benson 1. To summarize, we have:

Lemma 2.6. Let $d_{n}(p)$ be the dimension of a modular basic representation of $\tilde{A}_{n}$.

(i) Let $p>2$ and $p \nmid n$. Then $d_{n}(p)=2^{(n-3) / 2}$ if $n$ is odd, and $2^{(n-2) / 2}$ if $n$ is even.

(ii) Let $p>2$ and $p \mid n$. Then $d_{n}(p)=2^{(n-3) / 2}$ if $n$ is odd, and $2^{(n-4) / 2}$ if $n$ is even.

(iii) Let $p=2$. Then $d_{n}(2)=2^{(n-3) / 2}$ if $n$ is odd, $2^{(n-2) / 2}$ if $n \equiv 2(\bmod 4)$, and $2^{(n-4) / 2}$ if $n \equiv 0(\bmod 4)$.

We cite another result of Wales for future reference:

Proposition 2.7. Let $n>5$ and let $\phi$ be a faithful irreducible $r$ of $\tilde{A}_{n}$. Then $\phi$ is basic if and only if all composition factors of $\phi \mid \tilde{A}_{n-1}$ are basic.

Proof. For $\tilde{S}_{n}$ a similar result is contained in the proof of [11, Theorem 8.1]. Then Clifford theory implies the result for $\tilde{A}_{n}$.

Finally, we record a lemma of G. Higman which is often used below.

Lemma 2.8 ([2, Ch. IX, Theorem 1.10]). Let $G \subset G L(n, F)$ be a finite subgroup with abelian normal subgroup $A$ of order coprime to $p$. Let $g \in G$ be an element of order $p^{k}$ such that $g^{p^{k-1}} \notin C_{G}(A)$. Then $\operatorname{deg} g=p^{k}$.

\section{MAin RESUlts}

The following result of the second author provides us with an induction base for future arguments:

Lemma 3.1 ([12, Lemma 2.12]). Let $n<2 p$, let $G$ be a quasi-simple group with $G / Z(G) \cong A_{n}$, and let $g \in G$ be an element with $g^{p} \in Z(G)$. Suppose that $\phi$ is a faithful irreducible representation of $G$ such that $\operatorname{deg} \phi(g)<p$. Then one of the following holds:

(i) $Z(G)=1, n=p$, and $\phi=\varepsilon_{n}$ with $\operatorname{dim} \phi=p-2$;

(ii) $p=3, G=\tilde{A}_{5}$, and $\operatorname{dim} \phi=2$;

(iii) either $p=5, G \cong \tilde{A}_{6}$, or $p=5,7, G \cong \tilde{A}_{7}$, and in both cases $\operatorname{dim} \phi=4$;

(iv) $p=5, G=\tilde{A}_{8}$ or $\tilde{A}_{9}$, and $\operatorname{dim} \phi=8$;

(v) $p=5, G=\tilde{A}_{5}$, and $\operatorname{dim} \phi=2$;

(vi) $p=5, G=\tilde{A}_{5}$, and $\operatorname{dim} \phi=4$;

(vii) $p=5, G=3 \cdot A_{6}$ or $3 \cdot A_{7}$, and $\operatorname{dim} \phi=3$;

(viii) $p=7, G=3 \cdot A_{7}$ or $6 \cdot A_{7}$, and $\operatorname{dim} \phi=6$.

Moreover, in all the cases above, except (iv), the Jordan normal form of $\phi(g)$ has a single block, and in case (iv) it has two blocks of size 4.

Remark. The representations $\phi$ appearing in (ii) $-(\mathrm{v})$ are basic. 
Lemma 3.2. Let $G=A_{n}$ or $\tilde{A}_{n}$, with $n \geq 2 p>6$, and let $g \in G$ be an element of order $p$. If $p=5$, suppose additionally that $\pi(g)$ is a 5-cycle. If $p=7$ suppose additionally that either $G=A_{n}$ or $\pi(g)$ is a 7-cycle. If $\phi$ is a faithful irreducible representation of $G$ with $\operatorname{deg} \phi(g)<p$, then $p=5, G=\tilde{A}_{n}$, and $\phi$ is basic.

Proof. We may assume that $\pi(g)$ is a product of cycles of the form:

$$
\pi(g)=(1,2, \ldots, p)(p+1, \ldots, 2 p) \cdots .
$$

Recall that for $m<n, A_{m}$ is assumed to be embedded into $A_{n}$ as acting on the first $m$ letters, unless otherwise stated. Define a subgroup $H$ of $G$ by requiring that (1) $H \supset Z(G) ;(2) \pi(H) \cong A_{7}$ if $p=5 ; \pi(H) \cong A_{8}$ if $p=7$ and $G=\tilde{A}_{n} ; \pi(H) \cong A_{p}$ otherwise.

Set $X=\langle g, H\rangle$. Then we have $H \cong X / O_{p}(X)$ and $g=h g_{1}$, where $h=$ $(1,2, \ldots, p) \in H$ and $g_{1} \in O_{p}(X)$. Let $\tau$ be a nontrivial composition factor of $\phi \mid X$. Then $\tau\left(O_{p}(X)\right)=\mathrm{Id}$; so we can also consider $\tau$ as a representation of $H$. We have $\tau(g)=\tau(h)$. In view of Lemma $2.1 \operatorname{deg} \tau(g)<p$.

If $Z(G)=\{1\}$, then $Z(H)=\{1\}$, and so $\tau=\varepsilon_{n}$, thanks to Lemma 3.1. By induction on $n$ it follows from Corollary 2.4 that $\phi=\varepsilon_{n}$. The result now follows from Lemma 2.2.

Finally, let $|Z(G)|=2$. By Lemma 3.1, $p=5$ and $\tau$ is basic. So Proposition 2.7 implies that $\phi$ is basic.

Lemma 3.3. Let $G=\tilde{A}_{n}$ or $A_{n}$, and let $g \in G$ be an element of order $p>3$ such that $\pi(g)$ has $k$ nontrivial cycles. If $\operatorname{deg} \phi(g)<p$ for some faithful irreducible representation $\phi$ of $G$, then $k<3$.

Proof. Suppose $k \geq 3$. We may assume that

$$
\pi(g)=(1,2, \ldots, p)(p+1, p+2, \ldots, 2 p)(2 p+1,2 p+2, \ldots, 3 p) \ldots .
$$

Let $A$ be the elementary abelian 3 -subgroup of $A_{n}$ of order $3^{p}$ generated by the commuting 3-cycles $(j, p+j, 2 p+j)$ for $1 \leq j \leq p$. If $G=\tilde{A}_{n}$, let $B=\pi^{-1}(A)$. If $G=A_{n}$, take $B=A$. In both cases $B$ is abelian of order prime to $p$, and $g \in N_{G}(B) \backslash C_{G}(B)$. Now we apply Lemma 2.8

Lemma 3.4. Let $G=\tilde{A}_{n}$ or $A_{n}$, and let $g \in G$ be an element of order $p=5$ or 7 . If $\operatorname{deg} \phi(g)<p$ for some faithful irreducible representation $\phi$ of $G$, then $\pi(g)$ is a p-cycle.

Proof. In view of Lemma 3.3, we may assume that

$$
\pi(g)=(1,2, \ldots, p)(p+1, p+2, \ldots, 2 p) .
$$

Set $h_{i j}=(i, i+p)(j, j+p) \in A_{n}$ for $1 \leq i<j \leq p$. The subgroup $H$ generated by the $h_{i j}$ is abelian of order $2^{p-1}$. If $G=A_{n}$, we may apply Lemma [2.8, since $g \in N_{G}(H) \backslash C_{G}(H)$. Now, let $G=\tilde{A}_{n}$.

Assume first that $p=7$. Observe that $H$ can be considered as an $\mathbb{F}_{2}\langle\pi(g)\rangle$ module via conjugation, and $\langle\pi(g)\rangle$ is a cyclic group of order $p$. Then the dimension of $H$ over $\mathbb{F}_{2}$ is 6 . Hence $\langle\pi(g)\rangle$ has an irreducible constituent $M$ on $H$ of dimension 3. In other words, $\pi(g)$ normalizes $M$, and $[\pi(g), M] \neq 1$. Let $L=\pi^{-1}(M)$. Then $|L|=16$; hence it is not extraspecial. Now it is easy to deduce, using conjugation with $g$, that $L$ is abelian. Since $g \in N_{G}(L) \backslash C_{G}(L)$, the result follows from Lemma 2.8. 
Finally, let $p=5$. Then $g$ is contained in a group $X$ isomorphic to the central product of two copies of $\tilde{A}_{5}$. Let $\tau$ be an irreducible constituent of the restriction $\phi$ to $X$. Then $\tau=\tau_{1} \otimes \tau_{2}$ where $\tau_{1}$ and $\tau_{2}$ are faithful representations of the respective copies of $\tilde{A}_{5}$. In view of Lemma 3.1 and [5, Chapter VIII, Theorem 2.7], $\operatorname{deg} \tau(g)<5$ only if $\operatorname{dim} \tau_{1}=\operatorname{dim} \tau_{2}=2$. This means that every irreducible constituent of the restriction of $\phi$ to the naturally embedded $\tilde{A}_{5}$ is basic. By Proposition 2.7] $\phi$ is basic. Then $\operatorname{deg} \phi(g)=5$ by [8, Lemma 3.12].

Proof of Theorem 1.1] For $p=3$, see Chermak [3], and for $n<2 p$, see Lemma 3.1 Let $p>3$ and $n \geq 2 p$. Then the "only-if" part follows from Lemmas 3.2 3.4, For the "if" part it remains to show that $d:=\operatorname{deg} \phi(g)=4$ for $\phi$ basic spin, $p=5$, and $\pi(g)$ a 5 -cycle. Restricting to a natural subgroup $\tilde{A}_{7}$ containing $g$ and using Lemma 3.1 we see that $d \geq 4$. On the other hand, for complex representations of $\tilde{A}_{n}$ a theorem similar to Theorem 1.1 has been proved in [13. In particular, if $g \in \tilde{A}_{n}$ is a 5 -cycle, then $\operatorname{deg} \beta(g)=4$ for complex basic spin representations $\beta$. Since $\phi$ is a constituent of a reduction of $\beta$ modulo 5 , we have $d \leq 4$.

Now we prove Theorem 1.2 The result is contained in Lemmas 3.5 3.11

Lemma 3.5. Theorem 1.2 is true for $n=5$.

Proof. Since $A_{5}$ has no elements of order 4 we may assume that $G=S_{5}$. Then $G$ has two nontrivial irreducible representations, both of dimension 4; see [6, Tables]. One of them is $\varepsilon_{5}$, for which $\varepsilon_{5} \oplus 1_{S_{5}}=\pi$, where $\pi$ is the natural permutation representation of dimension 5. Clearly, Jord $\pi(g)=\operatorname{diag}\left(J_{4}, J_{1}\right)$; so Jord $\varepsilon_{n}(g)=J_{4}$. Another irreducible representation of $G$ corresponds to the partition $(3,2)$, and so it is reducible on $A_{5}$, thanks to [1] or [9]. Therefore, $\operatorname{Jord} \phi\left(g^{2}\right)=\operatorname{diag}\left(J_{2}, J_{2}\right)$ whence $\operatorname{Jord} \phi(g)=J_{4}$.

Lemma 3.6. Let $n \geq 5, G \in\left\{A_{n}, S_{n}, 3 \cdot A_{6}, 3 \cdot S_{6}, 3 \cdot A_{7}, 3 \cdot S_{7}\right\}$, and let $g \in G$ be an element of order 4 fixing at least one point of the natural permutation set. Then $\operatorname{deg} \phi(g)=4$ for any faithful irreducible representation $\phi$ of $G$.

Proof. We may assume that $g$ transitively permutes $1,2,3,4$ and fixes 5 . Let $H:=\operatorname{Alt}\{1,2,3,4,5\}$, and let $\hat{H}$ be the preimage of $H$ in $G$. Set $X:=\langle g, \hat{H}\rangle$. Since $H$ contains no element of order 4 , the restriction homomorphism $h: X \rightarrow$ $\operatorname{Sym}\{1,2,3,4,5\} \cong S_{5}$ is surjective. Let $K=\operatorname{ker} h$. Clearly, $K$ is central in $X$. Since $S_{5}$ has no non-split central extension with center of order 3 , we have $X \cong Z(G) \times Y$ for some subgroup $Y$ with $g \in Y$. Let $\tau$ be a composition factor of $\phi \mid Y$ with $\operatorname{dim} \tau>1$. Then $\tau(Y) \cong S_{5}$. By Lemma 3.5, $\operatorname{deg} \tau(g)=4$; hence $\operatorname{deg} \phi(g)=4$ in view of Lemma 2.1.

Lemma 3.7. Theorem 1.2 is true for $G=A_{6}$ and $S_{6}$.

Proof. For $g \in S_{6} \backslash A_{6}$ this follows from Lemma 3.6. So we may assume that $G=A_{6}$. We use [9]. Irreducible $F G$-modules of dimension 8 are projective. So the Jordan form of $g$ on each of these modules is $\operatorname{diag}\left(J_{4}, J_{4}\right)$. Other nontrivial irreducible $F G$-modules are of dimension 4. Since $A_{6} \subseteq S_{6} \cong S p(4,2)$, one of them is the natural $S p(4,2)$-module $V$ restricted to $A_{6}$. Since the Jordan form of a unipotent element of $S p(4,2)$ does not have a block of size 3, the theorem is true for the natural representation. The second $F G$-module of dimension 4 is obtained from $V$ by twisting with the outer automorphism $\sigma$ of $S_{6}=S p(4,2)$. Since $A_{6}$ has 
only one conjugacy class of elements of order $4, \sigma(g)$ is conjugate to $g$ in $A_{6}$; so Jord $\sigma(g)=$ Jord $g$.

Lemma 3.8. Let $n \geq 6, G=A_{n}$ or $S_{n}$, and let $g \in G$ be an element of order 4 having a 2-cycle in its cycle type. Then $\operatorname{deg} \phi(g)=4$ for any faithful irreducible representation $\phi$ of $G$.

Proof. Clearly $g$ normalizes a subgroup $H \cong A_{6}$ fixing $n-6$ points such that $g$ has a 2 - and 4 -cycle on the remaining 6 points. Then $g=g_{1} g_{2}$ where $g_{1} \in H$, $g_{2} \in C_{G}(H)$. Set $X=\langle g, H\rangle=\left\langle g_{2}, H\right\rangle$, and let $\tau$ be a nontrivial composition factor of $\phi \mid X$. Since $X / O_{2}(X) \cong H$, Lemma 3.7 gives $\operatorname{deg} \tau(g)=4$. So by Lemma 2.1, $\operatorname{deg} \phi(g)=4$.

Lemma 3.9. Theorem 1.2 is true for $n=8$.

Proof. In view of Lemmas 3.8 and 3.6, we may assume that the cycle type of $g$ is $(4,4)$ and $G=A_{8} \cong S L(4,2)$. Note that the group $A_{8}$ has 2 conjugacy classes of elements of order 4 , corresponding to cycle types $(4,2)$ and $(4,4)$, and only the first one meets the subgroup $A_{6}$. The group $S L(4,2)$ has 2 conjugacy classes of elements of order 4 , with Jordan forms $J_{4}$ and $\operatorname{diag}\left(J_{3}, J_{1}\right)$, and the second one does not meet $S p(4,2)$. Since $A_{6} \cong S p(4,2)^{\prime}$, we conclude that the class $(4,4)$ corresponds to the class $\operatorname{diag}\left(J_{3}, J_{1}\right)$. So $g$ belongs to the intermediate subgroup $H \cong S L(3,2)$.

Let $\tau$ be an irreducible representation of $H$. Then $\tau$ is a restriction of a rational representation of $\bar{H}$, the algebraic group of type $A_{2}$. The irreducible representations of $\bar{H}$ are labelled by their highest weights $a_{1} \omega_{1}+a_{2} \omega_{2}$, where $a_{1}, a_{2}$ are nonnegative integers and $\omega_{1}, \omega_{2}$ are the fundamental weights. It is well known that $\tau$ is a restriction of one of the four irreducible representations of $\bar{H}$ labelled by $0, \omega_{1}, \omega_{2}$, or $\omega_{1}+\omega_{2}$. The last one corresponds to the Steinberg module, whose restriction to $H$ is projective, and so all Jordan blocks of $g$ are of size 4. Two other representations are the natural and its dual. So the Jordan form of $g$ on both of them is $\operatorname{diag}\left(J_{3}, J_{1}\right)$. Finally, corresponding to the zero highest weight we have the trivial representation.

Now, let $\lambda=a_{1} \omega_{1}+a_{2} \omega_{2}+a_{3} \omega_{3}$ be the highest weight of $\phi$. By a theorem of Smith [10] (also proved independently by R. Dipper), the restriction $\phi \mid H$ contains a direct summand $\tau$ with highest weight $a_{1} \omega_{1}+a_{2} \omega_{2}$. From the previous paragraph, we may assume that at least one of $a_{1}, a_{2}$ is zero. By duality, the same is true for $a_{2}, a_{3}$. So we are left with the cases $\lambda \in\left\{\omega_{1}, \omega_{2}, \omega_{3}, \omega_{1}+\omega_{3}\right\}$. The last one is the adjoint representation of $G$. Clearly, its restriction to $H$ contains a composition factor isomorphic to the adjoint representation of $H$. Since the last representation is projective, it is a direct summand. Hence this case is ruled out. The cases $\lambda=\omega_{1}, \omega_{3}$ are obvious. Finally, the module corresponding to $\lambda=\omega_{2}$ is the exterior square of the natural module. So its restriction to $H$ is a direct sum of the natural and dual natural modules; hence the Jordan blocks of $\phi(g)$ are of size 3 .

Lemma 3.10. Let $G=A_{n}$ or $S_{n}$, and let $g \in G$ be an element of order 4 containing at least three 4 -cycles. Then $\operatorname{deg} \phi(g)=4$ for any faithful irreducible representation $\phi$ of $G$.

Proof. We may assume that

$$
g=(1,2,3,4)(5,6,7,8)(9,10,11,12) \ldots
$$


Set $h_{j}:=(j, j+4, j+8)$ for $j=1,2,3,4$, and $H:=\left\langle h_{1}, h_{2}, h_{3}, h_{4}\right\rangle$. Then $H$ is an abelian 3-group and $g \in N_{G}(H)$. Moreover, $g^{2} \notin C_{G}(H)$; so the result follows from Lemma 2.8

Lemma 3.11. Theorem 1.2 is true for $G=3 \cdot A_{6}$ and $3 \cdot A_{7}$.

Proof. For $G=3 \cdot A_{7}$ see Lemma 3.6. Let $G=3 \cdot A_{6}$. Then $\operatorname{dim} \phi=3$ or 9 ; see 9]. In the former case, $\operatorname{deg} \phi(g)=3$, since $\operatorname{deg} \phi(g)<3$ implies $\phi(g)^{2}=1$, which is false. Let $\operatorname{dim} \phi=9$. Observe that $g^{2}$ normalizes a cyclic group $\langle c\rangle$ of order 5 . Set $X:=\left\langle g^{2}, c\right\rangle$. Since $g^{2} c g^{-2}=c^{-1}$ and the multiplicity of every eigenvalue $\alpha \neq 1$ of $\phi(c)$ is 2 (see [9]), it follows that $\phi \mid X$ has four composition factors of dimension 2 and one composition factor of dimension 1 . Therefore $\operatorname{Jord} g^{2}=\operatorname{diag}\left(J_{2}, J_{2}, J_{2}, J_{2}, J_{1}\right)$, whence $\operatorname{Jord} g=\operatorname{diag}\left(J_{4}, J_{4}, J_{1}\right)$.

\section{REFERENCES}

[1] D. Benson, Spin modules for symmetric groups, J. London Math. Soc. (2) 38 (1988), 250-262. MR 89k:20020

[2] B. Huppert and N. Blackburn, Finite groups II, Grundlehren der mathematischen Wissenschaften, Band 242, Springer-Verlag, Berlin, 1982. MR 84i:20001a

[3] A. Chermak, Quadratic Pairs, preprint, 2001.

[4] Ch. Curtis and I. Reiner, Representation theory of finite groups and associative algebras, John Wiley and Sons, New York-London, 1962. MR 26:2519]

[5] W. Feit, The representation theory of finite groups, North-Holland, Amsterdam, 1982. MR 83g:20001

[6] G. D. James, The Representation Theory of the Symmetric Groups, Lecture Notes in Mathematics, Vol. 682, Springer-Verlag, Berlin-New York, 1978. MR 80g:20019

[7] A. S. Kleshchev, Branching rules for modular representations of symmetric groups, II, J. reine angew. Math. 459 (1995), 163-212. MR 96m:20019b

[8] A. S. Kondratiev and A. E. Zalesski, Linear groups of degree at most 27 over residue rings modulo $p^{k}$, J. Algebra 240 (2001), 120-142. MR 2002c:20076

[9] C. Jansen, K. Lux, R. A. Parker, and R. A. Wilson, An atlas of Brauer characters, The Clarendon Press, Oxford University Press, New York, 1995. MR 96k:20016

[10] S. D. Smith, Irreducible modules and parabolic subgroups, J. Algebra 75 (1982), 286-289. MR 83g:20043

[11] D. Wales, Some projective representations of $S_{n}$, J. Algebra 61 (1979), 37-57. MR 81f:20015

[12] A. E. Zalesskiı̌, Minimal polynomials and eigenvalues of p-elements in representations of quasi-simple groups with a cyclic Sylow p-subgroup, J. London Math. Soc. (2) 59 (1999), 845-866. MR 2001a:20018

[13] A. E. Zalesskiř, Eigenvalues of prime-order elements in projective representations of alternating groups, Vestsi Akad. Navuk Belarusi, ser. Fiz.-Mat-Inform. (1996), no. 3, 41-43 (in Russian). MR 98b:20017

Department of Mathematics, University of Oregon, Eugene, Oregon 97403

E-mail address: klesh@math.uoregon.edu

School of Mathematics, University of East Anglia, Norwich NR4 7TJ, England

E-mail address: a.zalesskii@uea.ac.uk 